\title{
Temporal interval production and short-term memory
}

\author{
DAVID T. FIELD \\ University of Reading, Reading, England \\ and \\ JOHN A. GROEGER \\ University of Surrey, Guildford, England
}

\begin{abstract}
Interference with time estimation from concurrent nontemporal processing has been shown to depend on the short-term memory requirements of the concurrent task (Fortin \& Breton, 1995; Fortin, Rousseau, Bourque, \& Kirouac, 1993). In particular, it has been claimed that active processing of information in short-term memory produces interference, whereas simply maintaining information does not. Here, four experiments are reported in which subjects were trained to produce a 2,500-msec interval and then perform concurrent memory tasks. Interference with timing was demonstrated for concurrent memory tasks involving only maintenance. In one experiment, increasing set size in a pitch memory task systematically lengthened temporal production. Two further experiments suggested that this was due to a specific interaction between the short-term memory requirements of the pitch task and those of temporal production. In the final experiment, subjects performed temporal production while concurrently remembering the durations of a set of tones. Interference with interval production was comparable to that produced by the pitch memory task. Results are discussed in terms of a pacemaker-counter model of temporal processing, in which the counter component is supported by short-term memory.
\end{abstract}

One popular account of temporal judgment suggests that the brain possesses an internal clock. The function of the clock is achieved by the interaction of four components. A pacemaker generates pulses at a fixed mean rate, and a short-term memory system operates as a counter of the pulses. Also proposed is a long-term store, which retains information about important durations, represented as numbers of pulses. Finally, a comparison process compares the current value in the counter with the relevant values in the long-term store and signals when they are equal. For examples of specific implementations of internal clock theory, see Fetterman and Killeen (1990) or Gibbon, Church, and Meck (1984).

One question about the internal clock system is what cognitive resources are required to use it to make a temporal judgment. This question has been addressed by requiring subjects to perform temporal judgments while they simultaneously perform a secondary nontemporal task. In a temporal reproduction task, in which subjects are first presented with a target interval and then attempt to reproduce it as accurately as possible, a secondary task per-

Correspondence concerning this article should be addressed to D.T. Field, Department of Psychology, University of Reading, Reading RG6 6AL, England (e-mail: d.t.field@rdg.ac.uk).

Note-This article was accepted by the previous editorial team, headed by Neil Macmillan. formed when the target interval is being reproduced lengthens the reproduced interval (e.g., Fortin \& Rousseau, 1998). However, when the secondary task is performed instead during the presentation of the target interval, the reproduced interval is shortened. Both of these findings can be explained by assuming that the secondary task interrupts the accumulation of pulses in the counter. If the secondary task occurs during the presentation of the target interval, the number of pulses representing the interval is reduced, and when this representation is used as the target for the subsequent reproduction phase, an objectively short reproduction results. If the secondary task is performed during the reproduction phase, the number of pulses representing the target interval is normal, but the rate at which pulses accumulate to satisfy the target is reduced, resulting in an objectively long reproduction. This basic pattern of results has been reported using various secondary tasks by various authors, including Hicks, Miller, and Kinsbourne (1976), Brown and West (1990), Casini and Macar (1997), and Zakay (1993). Typically, as the difficulty of the secondary task increases, the biasing observed in the time estimation task grows. The secondary tasks used have included card sorting, manual tracking, and light intensity discrimination. The generality of results obtained with such diverse secondary tasks led Zakay (1989) to suggest that any secondary task processing interferes with temporal judgment. Zakay (1989) further suggested that the cognitive resource required to operate the pacemaker-counter mechanism is attention, conceived of as a general pur- 
pose modality-free processor. Attention may operate as a "gate," interposed between the pacemaker and the counter (Zakay \& Block, 1997), which operates like an electrical circuit, allowing pulses through when it is closed. In this model, attentional resources are required to keep the gate in its closed state. Secondary task processing diverts attentional resources elsewhere, causing the gate to open, so that no (or possibly fewer) pulses may pass from the pacemaker to the counter.

However, Fortin, Rousseau, Bourque, and Kirouac (1993) observed that secondary tasks combined with temporal judgment have typically been complex ones, making requirements upon a number of cognitive subsystems simultaneously. Therefore, when the difficulty of these tasks is increased, perceptual, motor, and/or memory requirements may change concurrently. For example, there were three difficulty levels of the cardsorting task used by Hicks et al. (1976). In the easiest condition, a deck of cards was dealt into a single stack. In another condition, the deck was dealt into two stacks according to color, whereas in the hardest condition, it was dealt into four stacks according to suit. As the difficulty of this task increases, the motor component of the task becomes more demanding, as does the required perceptual judgment and the complexity of the memory processes required to support it. Because of this, Zakay's (1989) argument concerning the generality of interference between temporal judgment and secondary task is not strongly supported by the available data. Rather, it is possible that the observed interference arises from a subset of the cognitive requirements of the secondary task.

Fortin et al. (1993) investigated this possibility by exercising more precise control over the processing resources required by the secondary tasks performed concurrently with temporal judgment. In order to achieve this control, they developed a paradigm in which the perceptual and motor requirements of the nontemporal component of the dual task were kept minimal and constant, while the information-processing requirements were systematically varied. The procedure, called interval production, which is similar to that used here, involved training a subject to consistently produce a target interval of 2,000 msec, the beginning and end of which were indicated by the subject's pressing a key. Once this task was mastered, the memory probe stimulus or visual stimulus array for various search tasks was presented during interval production, and the yes/no response to the search task was also used by the subject to indicate the end of interval production. Set size with interval production slopes for each secondary task were compared with each other, as well as with the set size slopes for the same tasks under reaction time conditions.

Fortin et al. (1993) used this method to show that interference with time estimation is not due to the nonspecific attentional requirements of a secondary task. Instead, they argued that the magnitude of interference is determined by the short-term memory requirements of a secondary task. They reported four interval production experiments, in which they attempted to keep secondary task difficulty (as indexed by reaction time with set size slopes) constant, while systematically varying the amount of short-term memory resources required by the secondary tasks. When the secondary task was the Sternberg (1966) memory search task, requiring the retention of between one and six digits in short-term memory, which are then scanned for the presence or absence of a single probe item, the slope of set size with interval production was greatest. In a second condition, there was always just one item to remember, and the subjects had to search for the presence or absence of that item in a display composed of multiple items. The slope of interval production with the number of items in the display was less than that in the Sternberg experiment, even though the reaction time slopes for the two tasks were comparable. The authors argued that this occurred because of the relative reduction in short-term memory load in the second task. In two more experiments, memory load was made as low as possible by employing a feature-absent visual search task and varying the number of items in the display. In this condition, the subjects looked for the same thing in every trial (which was the absence of a small component in one of a number of otherwise identical visual items). These tasks produced the most substantial slopes under reaction time conditions but no slope at all with concurrent interval production. The results of these experiments have two main implications. First, because featureabsent visual search did not affect concurrent interval production, they demonstrate that not all secondary task processing interferes with temporal processing, indicating that the cognitive resources required by temporal processing are specific, rather than general, in nature. Second, because the amount of interference with interval production caused by a secondary task was proportional to the short-term memory requirements of the task, the authors suggested that short-term memory is a critical resource for timing. In terms of the pacemaker-counter model, the gating process might be under the control of short-term memory. When short-term memory resources are not available, the gate may not be fully closed, resulting in a reduced rate of accumulation of pulses in the counter.

Brown (1997) provided further evidence against the simplest form of the attentional allocation model. Brown's experiments were designed to allow the impact of timing upon the secondary task to be assessed, as well as the more usual impact of the secondary task upon timing. Pursuit rotor tracking, visual search, and mental arithmetic all interfered with timing, but timing disrupted only mental arithmetic. Brown argued that processing resources are specific, rather than general, and his results are clearly inconsistent with the more simple formulation of Zakay (1989).

More recently, Fortin and Breton (1995) identified two possible sources of interference with temporal production that might be induced by the concurrent Sternberg memory search task: disruption due to the process 
of comparing the probe item with the items in memory, and disruption due to the requirement to maintain the items through the first part of interval production before probe presentation. To distinguish between these two possibilities, they altered the paradigm to isolate the maintenance component of the memory search task. The memory set was now maintained throughout interval production, the probe item being presented once interval production was complete. If the requirements of maintaining the memory set involved sharing resources with the pacemaker-counter system, a set size with interval production slope would occur. Results indicated that there was no slope with memory set size, which Fortin and Breton took to suggest that the interference with temporal processing observed by Fortin et al. (1993) was due to the active process of comparing the probe item with each of the items in short-term memory. This conclusion was supported by two further experiments in which active processing in short-term memory was required, without the need to maintain information over time. In one experiment, the amount of active processing was manipulated by increasing the number of syllables in a rhyme judgment task, and in a second experiment, the number of degrees of mental rotation of a geometric shape was manipulated. Both these experiments indicated that interval production was lengthened by the presence of the secondary task, by an amount that was proportional to the duration of secondary task processing, as measured in reaction time control conditions.

Fortin and colleagues' results have clearly demonstrated that the cognitive resources required by the pacemakercounter mechanism are specific, rather than general. This follows from the fact that they have been able to devise secondary tasks for which increasing the difficulty of the secondary task has no effect on temporal processing. The aim of the present research was to further investigate Fortin and colleagues' claim that only active processing of material in short-term memory produces systematic interference with interval production. In Fortin and Breton (1995), this claim rests upon a single demonstration that increasing the size of a memory load consisting of digits does not result in a set size effect on interval production. Here, this experiment was extended, using four qualitatively different kinds of memoranda. In two of the four cases, a set size effect was obtained, suggesting that the relationship between short-term memory and temporal processing is more complex than previously suggested.

\section{EXPERIMENT 1}

The memory set used by Fortin and Breton (1995) in their memory load only plus interval production experiment consisted of visually presented digits. Increasing the number of digits to be remembered had no effect upon interval production. There is considerable evidence that visually presented digits are retained in a phonological store within short-term memory (e.g., Baddeley,
1986). The aim of Experiment 1 was to extend this experiment, using memory material known to depend on a subsystem of short-term memory other than the phonological store, and by doing so to test the generality of Fortin and Breton's findings.

The present experiment required subjects to perform a pitch memory task concurrently with interval production. Pitch was chosen because it has been dissociated from phonological material in short-term memory. For example, Deutsch (1970) demonstrated that recognition memory for a standard tone was considerably degraded by the subsequent presentation of other tones, but not by the subsequent presentation of speech, even if the speech had to be processed. Deutsch (1970) proposed a separate store for pitch and, in further research, mapped out the characteristics of the store (Deutsch, 1972, 1975; Deutsch $\&$ Feroe, 1975). More recently, several cases of amusia without aphasia have been reported (Peretz, Belleville, \& Fontaine, 1997; Peretz et al., 1994). In amusia without aphasia, language processing and the processing of environmental sounds are preserved, whereas perception and memory for basic aspects of music, such as pitch and melody, are impaired. This dissociation supports the proposition that pitch information and phonological information, such as the digits used in Fortin and Breton's (1995) secondary task, are retained in different shortterm stores.

Cruse and Jones (1976) have reported a pitch analogue of the Sternberg task, and this was adopted for use as a secondary task here. The stimuli consisted of eight perceptually equidistant pure tones, none of which corresponded to notes on the Western musical scale. On each trial, between one and four tones were presented, followed by a probe item. Subjects had to indicate by a buttonpress whether the probe item was present in the memory set. In order to facilitate comparisons between the present experiment and those of Fortin and colleagues, three conditions were tested. These were the following: first, a reaction time condition for the pitch memory search; second, a condition in which the probe item for the memory search was presented during interval production; and third, the critical condition, in which the subjects were required only to remember pitch information during interval production.

\section{Method}

Subjects. Seven subjects participated in the reaction time condition. Thirteen subjects took part in the condition in which the memory probe item was presented during interval production. Twelve subjects participated in the condition in which only pitch memory load was concurrent with interval production. All the subjects were unpaid volunteers, and each subject participated in only one condition.

Memory search task. On each trial, a memory set of between one and four tones was presented sequentially to the subject. The memory set size varied randomly from trial to trial. Tones were selected randomly without replacement from the memory set on each trial. After a short interval, which varied between experimental conditions (see below), a probe item was presented, which required a positive response on $50 \%$ of the trials. Positive probe items were selected randomly from the current memory set. Negative probe items 
were randomly selected from those set members that were not part of the current memory set.

The memory set consisted of eight pure tones of 500-msec duration $(259,360,500,695,966,1343,1866$, and $2591 \mathrm{~Hz})$, presented using Hi-Tex CHP-727V headphones. Pauses between items in each memory set were also of 500-msec duration. Each tone had a frequency 1.38 times that of the previous one in the set. This resulted in roughly perceptually equal steps. The intensity level was set to slightly below the maximum comfortable level during piloting (approximately 70 decibels), and this level was maintained throughout the experiment.

Interval production task. The subjects in all the experimental conditions except the reaction time condition were first trained to consistently produce a $2,500-\mathrm{msec}$ interval. Interval production training began with the demonstration of the standard interval, which was presented as a visual stimulus. In order to perform a practice trial, those subjects who would go on to perform the task in which memory load was concurrent with interval production pressed the middle of three mouse buttons and waited for the duration they considered equal to the target before pressing the same button again. The situation was similar for those subjects who would go on to perform the memory probe during the interval production condition. The only difference was that an arrow appeared during each practice trial, indicating which of two buttons should be used to end the interval production. This modification maintained similarity between the practice and the experimental trials. In one kind of training procedure, feedback on the extent of over- or underestimation was given after each trial. An error of $10 \%$ or less in either direction was defined as a hit. Averaged feedback was given at the end of each block. Another kind of training involved only averaged feedback at the end of each block. Each subject received two blocks of 48 trials with full feedback, followed by two blocks of 24 trials with only endof-block feedback. The subjects were then introduced to the experimental task and performed 48 practice trials under those conditions. This was followed by a further two blocks of 24 interval production practice trials with full feedback and two blocks of 24 interval production trials with only end-of-block feedback.

Experimental conditions. In the reaction time condition, the last member of the memory set was followed immediately by a fixation dot. This was a signal to the subject to initiate the probe item by pressing the middle of three buttons; $500 \mathrm{msec}$ later, the probe tone was presented and continued to sound until a response had been made. The subjects pressed the left button to indicate a positive response and the right button to indicate a negative response. Feedback was given after each trial. Reaction time was measured from the onset of the probe item. The subjects were instructed to respond as quickly and accurately as possible. Trials were arranged in blocks of 48 , with three testing sessions, each of four blocks. The data from the last eight blocks were analyzed.

The sequence of events in the condition in which the memory probe item had to be processed during interval production was similar to that in the reaction time condition. After the memory set was presented, the subject pressed the middle button. This initiated interval production. After $500 \mathrm{msec}$ had elapsed, the probe item was presented and continued to sound until the subject's next buttonpress. This final buttonpress was made when the subject judged that time equal to the learned duration had elapsed since the first buttonpress. The final buttonpress was also used to indicate the response to the probe item, as in the reaction time condition. The subjects completed four blocks, each of which was composed of 48 trials.

In the final condition, the memory set items had to be remembered throughout interval production, and the probe item was presented after interval production was complete. Therefore, the sequence of events began with the presentation of the memory set. The subject initiated interval production by pressing the middle button and ended it with the same button. This buttonpress also caused
Table 1

Percentage Error Rates for the Pitch Memory Search Task in Experiment 1, Broken Down by Set Size and Probe Type, as Well as Experimental Condition

\begin{tabular}{lcrrr}
\hline & \multicolumn{4}{c}{ Set Size } \\
\cline { 2 - 5 } \multicolumn{1}{c}{ Condition } & \multicolumn{1}{c}{2} & \multicolumn{1}{c}{3} & \multicolumn{1}{c}{4} \\
\hline Positive probe reaction time & 1.2 & 5.1 & 13.7 & 19.0 \\
Negative probe reaction time & 4.5 & 5.1 & 9.8 & 8.3 \\
Positive probe during interval & 0.6 & 4.2 & 10.9 & 14.7 \\
Negative probe during interval & 2.9 & 5.4 & 8.7 & 12.5 \\
Positive probe after interval & 4.2 & 14.6 & 25.3 & 23.3 \\
Negative probe after interval & 9.0 & 14.9 & 21.2 & 28.1 \\
\hline
\end{tabular}

the probe item for the memory search task to be presented, requiring a final left or right buttonpress by the subject. In this condition, two functions were collected from each subject: interval production with set size and response time by set size and probe type (positive vs. negative). The subjects completed four blocks, each of which was composed of 48 trials.

\section{Results}

Data screening. Trials in which the response to the memory probe item was incorrect were excluded completely from further analysis. The overall error rate was $8.3 \%$ in the reaction time condition, as compared with $7.5 \%$ in the memory probe during interval production condition and $17.6 \%$ when the memory probe was presented after interval production. Error rates for each condition, broken down by memory set size and positive versus negative probe, may be found in Table 1 . In all three conditions, the error rate increased with set size, as is indicated in Table 1. In all the experiments reported here, increases in the error rate with set size were statistically reliable. After errors were excluded, outliers of more than three standard deviations were removed from each subject's data, for each experimental condition separately. This resulted in the removal of $1.6 \%$ of the remaining data in the reaction time condition and $0.7 \%$ in the interval production concurrent with the memory search

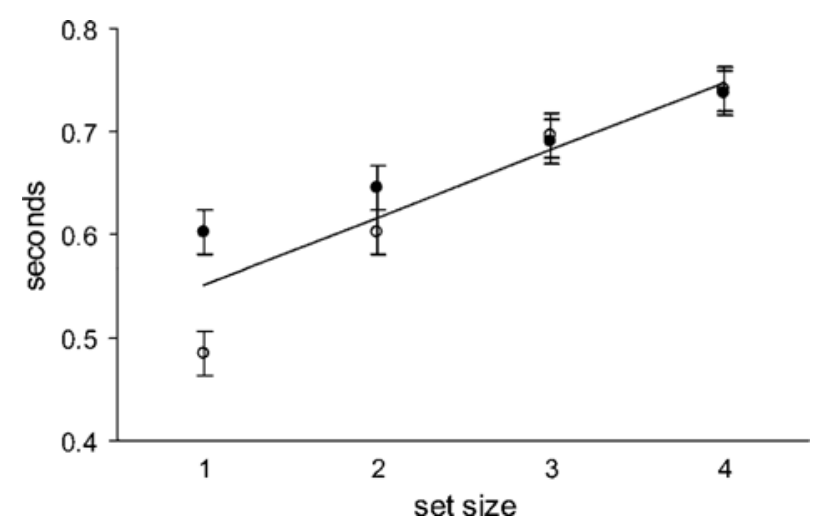

Figure 1. The effect on reaction time of set size and whether the probe item was present (open circles) or absent (filled circles) from the pitch memory set, together with the best linear fit to the data points. 
data condition. Where memory load only was concurrent with interval production, $0.5 \%$ of the interval data and $1.4 \%$ of the responses to the probe after interval production were classified as outlying.

Memory search reaction time alone. Figure 1 shows the effects of memory set size on reaction time. Linear regression indicated that the slope of set size with reaction time was $66 \mathrm{msec}$ per item. An analysis of variance was used to generate a polynomial contrast, enabling comparison of the linear, quadratic, and cubic fits to the set size with reaction time function. The linear component of the contrast provided the best fit $[F(1,6)=18.60$, $p<.01]$, indicating that linear regression was an appropriate method for calculating the slope of the function. This proved to be the case in all the experiments reported here, and so a regression slope and a linear contrast are provided in each case. The effect of set size interacted with probe type such that positive items were responded to more quickly at the lower set sizes only $[F(3,18)=$ 10.94, $p<.001]$.

Memory probe during interval production. In the final block of interval production practice without feedback, the mean interval produced was $2,765 \mathrm{msec}$, and the mean interval produced across the four experimental blocks as a whole $(2,627 \mathrm{msec})$ did not differ significantly from this. Figure 2 shows the effects of memory set size on interval production when the memory probe item was presented during interval production and the buttonpress indicating the response to the memory probe also ended the interval. Averaging across positive and negative trials, regression indicated that the slope in time production was $32 \mathrm{msec}$ per item. The linear contrast of set size with time production was significant $[F(1,12)=$ $6.54, p<.01]$, indicating that the slope was significant. The interaction of positive versus negative probe type with set size did not reach significance.

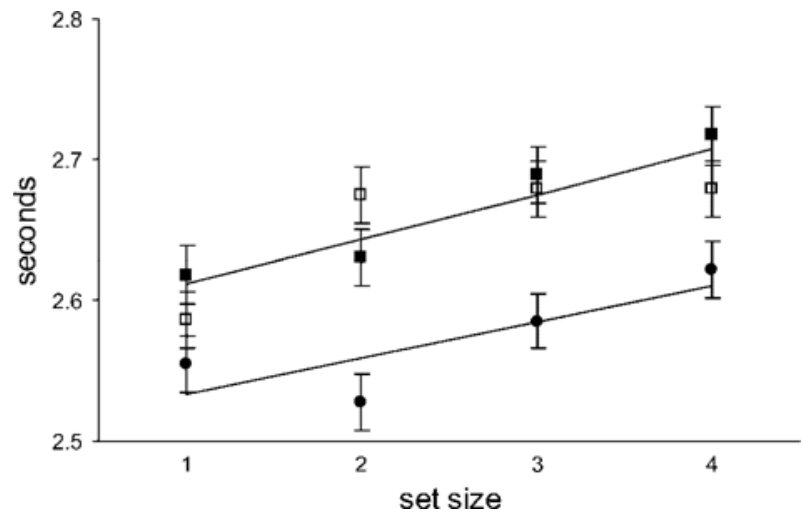

Figure 2. The data points clustered around the upper regression line are those for time production with concurrent pitch memory search. The probe-present condition is represented by open squares, and the probe-absent condition by closed squares. The filled circles are the data points for the condition in which the students had only to remember pitch information during time production.

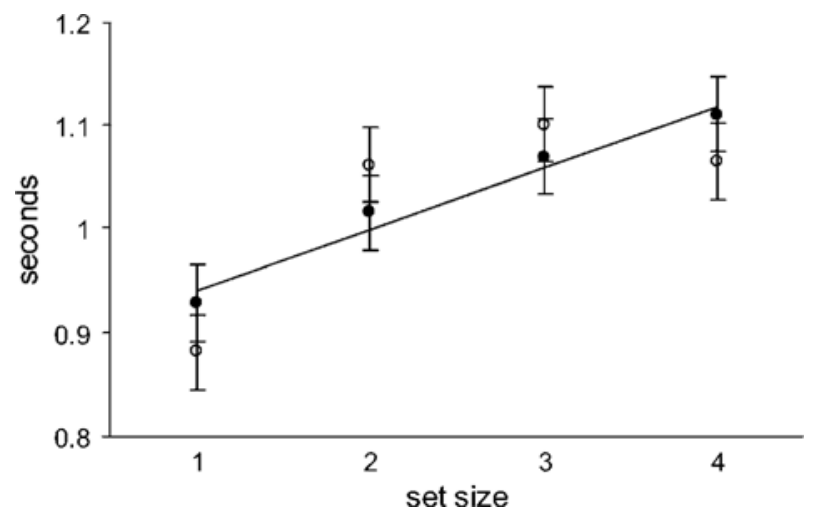

Figure 3. The effect of set size and whether the probe item was present (open circles) or absent (filled circles) from the pitch memory set on reaction time when the probe item was presented after time production was complete, together with the best linear fit to the data points.

Memory probe after interval production. In the final block of interval production practice without feedback, the mean interval produced was $2,700 \mathrm{msec}$. As in the other interval production condition, the mean interval produced across the four experimental blocks $(2,570 \mathrm{msec})$ did not differ significantly from that in the last practice block. This indicates that the introduction of secondary tasks did not dramatically disrupt, or qualitatively alter, the timing process. The effects observed are small but highly systematic, suggesting very specific interference. Figure 2 also shows the effects of memory set size on interval production when the memory probe item was presented after interval production. The subjects in this condition did not perform any search-related processing during interval production; however, it was necessary for them to retain the memory set throughout the interval. Regression indicated that the time production slope in this condition was $26 \mathrm{msec}$ per item. This slope, which was not predicted by Fortin's model of interference between short-term memory and interval production, produced a significant linear contrast $[F(1,11)=$ $6.54, p<.01]$. Figure 3 shows the response times to the memory probe, presented after interval production. As was expected, the linear contrast of set size with reaction time was significant $[F(3,33)=10.61, p<.01]$, there being no reliable interaction or difference between positive and negative trials. The regression slope of the function was $60 \mathrm{msec}$, as compared with $66 \mathrm{msec}$ in the reaction time condition.

\section{Discussion}

The initial aim of Experiment 1 was to extend Fortin and colleagues' findings concerning short-term memory and temporal processing by using a memory task dependent on a different module of short-term memory than were the phonological tasks used by Fortin. Fortin's main claim was that temporal processing is critically dependent on the availability of resources for active manipulation of material in short-term memory. The results of our 
first interval production experiment, in which the subjects were required to perform interval production concurrently with a pitch memory search, are consistent with Fortin's position. As the duration of memory search increased, interval production lengthened. The gate model can easily accommodate this result; the gate is forced to open due to lack of resources during the active memory search, causing a temporary interruption in the transmission of pulses from the pacemaker to the accumulator. However, the result of our second interval production condition, in which pitch information was maintained only during the production, is not consistent with Fortin's position or the gate model. As the number of items maintained increased, interval production lengthened, despite the absence of active manipulation of material in memory. When Fortin and Breton (1995) conducted a similar experiment with phonological material, no interference was found, which led them to conclude that active manipulation of material in short-term memory was a necessary condition for interference between temporal processing and a secondary task. Our results demonstrate that active manipulation of material in short-term memory is not a necessary condition for a secondary task to interfere with temporal processing. Furthermore, the gate model cannot account for our finding, because there is no portion of the interval to which the secondary task is localized. In the concurrent search and interval production condition, the approximate duration of the secondary task processes are known, and it is reasonable to suggest that the gate opens for this period and then closes again. In the memory load only condition, it is hard to see when the gate would be open and when it would be closed.

It might be argued that the different result for remembering pitch information than for remembering digits arises because of differences in task difficulty. Under reaction time conditions, the pitch memory search task yielded a slope of $66 \mathrm{msec}$ per item, as compared with $36 \mathrm{msec}$ for digit memory search, as had been found by Fortin et al. (1993) and many other researchers previously. When interval production was combined with pitch memory search, this resulted in a set size slope of $32 \mathrm{msec}$ per item, as compared with $24 \mathrm{msec}$ for the equivalent condition with digits (Fortin et al., 1993). These results suggest that the pitch memory task is more difficult (per item) under reaction time conditions than is the traditional digit-based Sternberg task. Perhaps this is why maintenance produced a set size effect only with pitch. In fact, Fortin and Massé (1999) have reported an experiment that renders the task difficulty explanation unlikely. They increased the difficulty of their phonologically based memory task by requiring subjects to retain and process the order, as well as the identity, of the items in the set, which was now made up of consonants. A digit indicating order accompanied the probe item, and the subjects had to verify that both the item and the order information was correct before making a positive response. This resulted in a large 100-msec slope under reaction time only conditions and a 57-msec slope when the probe item for this task was presented during interval production. Therefore, this task required greater processing resources per item than did the pitch task used here, but when Fortin and Massé (1999) used this task in the condition in which subjects had only to maintain the memory set during interval production, no set size slope was found. The combination of these results with those of Experiment 2 reported here indicates that difficulty level cannot explain the dissociation between the effects of maintaining digits and those of maintaining pitch on interval production.

The error rate generally increased with set size, which also was the case in the other experiments reported here. The implications of this are considered in the General Discussion section.

\section{EXPERIMENT 2}

Experiment 1 revealed a dissociation between the effects of retaining phonological material in short-term memory and the effects of retaining pitch on temporal processing. It is possible that one of these results represents a unique case. Alternatively, pitch and phonological material may each be members of distinct classes of memoranda, which differ in their effects on temporal judgment. To begin the task of distinguishing between these two possibilities, Experiments 2 and 3 replicated the conditions in Experiment 1, using different memory tasks.

Memory sets composed of phonological material, such as digits and consonants, are postcategorical because the sensory information making up the individual items is processed and identified with representations held in long-term memory before storage in short-term memory or memory search takes place. On the other hand, a pitch memory set consists of precategorical sensory information, and an infinite number of such memory sets can potentially be generated. As a "stimulus" to be retained in short-term memory, temporal information is also precategorical. Because both pitch and temporal information are precategorical, retaining them may require some resource that is not required by the retention of digits. This theory predicts that all memory tasks requiring the retention of precategorical sensory information will interfere with interval production, independently of difficulty level. To test this prediction, a memory set composed of different colors was created. In order to maintain the precategorical aspect of the task, all the colors were shades of gray, which prevented verbal labeling of the stimuli.

\section{Method}

Subjects. Eleven subjects, between 21 and 30 years of age, took part in the experiment. As an incentive, the 3 subjects with the lowest error rates were awarded cash prizes.

Stimuli. To create an appropriate analogue of the pitch memory set, eight shades of the color gray were used. Perceptual equidistance of each stimulus from the next in the series was ensured by using the Munsell color notation system. The Munsell notation sys- 
tem defines colors along the dimensions of value (brightness), chroma (saturation), and hue (dominant wavelength). Each of these attributes varies between 0 and 10 . In order to produce gray, chroma and hue were set to zero, and value was set to be greater than zero. The eight settings of value used were 1.1, 2.2 , 3.3, 4.4, 5.5, 6.6, 7.7, and 8.8. With these settings, the darkest and lightest grays were as far away from black and white as the members of the set were from each other. The stimuli were displayed as circles in the center of the computer monitor. The background consisted of many small gray squares. These were arranged in a new random pattern for each subject. The shades of gray used for the background were different from those in the memory set, but their mean Munsell value was equal to the mean Munsell value of the memory set. The values employed were 1.3, 2.4, 3.7, 4.6, 5.3, 6.4, 7.5, and 8.4.

Procedure. Each memory set item was presented for $1.5 \mathrm{sec}$, with no interval between successive items. Procedures for interval production training, the arrangement of experimental trials, the selection of memory sets, and probe items were identical to those used in the memory load condition of Experiment 1.

\section{Results}

The overall error rate in the memory search was $21.7 \%$, which was slightly higher than that for pitch. The details of this are presented in Table 2, from which it can be seen that the error rate increased with memory set size. After trials on which a memory search error occurred were excluded from the interval production and response time data, outlying data points were removed. These amounted to $2.18 \%$ of the memory search data and $0.97 \%$ of the interval production data.

In the final block of interval production practice without feedback, the mean interval produced was $2,530 \mathrm{msec}$, and the mean time produced in the experiment $(2,677 \mathrm{msec})$ did not differ significantly from this. Figure 4 shows the effect of increasing the number of items to remember on interval production. Regression indicated that the slope of the set size function was only $4 \mathrm{msec}$ per item. The linear contrast for this small slope was not significant $[F(1,10)=0.23$, n.s. $]$, and therefore, the hypothesis was not supported.

Figure 5 presents the response times to the memory probe, which was presented after interval production was complete. As was expected, the linear contrast of the effect of set size was significant $[F(1,10)=19.09, p<$ $.001]$, there being no reliable interaction or difference between positive and negative trials. The regression slope of the function was $110 \mathrm{msec}$, which was much greater than that for the pitch memory set.

\section{Discussion}

The relatively steep slope of the response time function to the memory probe suggests that this memory task

Table 2

Percentage Error Rates for the Color Memory Search Task in Experiment 2

\begin{tabular}{crccc}
\hline & \multicolumn{4}{c}{ Memory Set Size } \\
\cline { 2 - 5 } Probe Type & \multicolumn{1}{c}{1} & 2 & 3 & 4 \\
\hline Positive & 6.4 & 22.7 & 27.3 & 28.4 \\
Negative & 10.6 & 17.8 & 26.9 & 33.3 \\
\hline
\end{tabular}

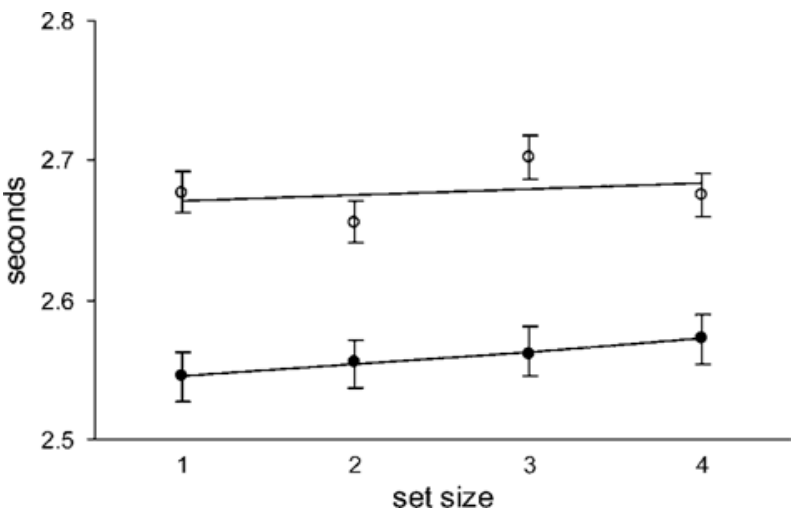

Figure 4. The data points (open circles) clustered around the upper regression line are those for time production with concurrent color memory load in Experiment 2. The filled circles are the data points for the condition in which the subjects had to remember timbre information during time production in Experiment 3.

was harder than the pitch memory task. Despite this, there was no set size effect of maintaining the memory set items on concurrent interval production, and neither was there an effect of secondary task versus no secondary task. This lack of interference stands in contrast to the interference obtained with the easier pitch memory task and supports the argument concerning task difficulty made in the Discussion section of Experiment 1.

The color memory task was designed to require the subjects to remember precategorical sensory information, in the hope that this would make it similar in its effects to the pitch memory task and dissimilar to the digit memory task used by Fortin and Breton (1995). However, the results of combining the color task with interval production were similar to those obtained with digits. Therefore, the distinction between memory for uncategorized sensory information and postcategorical phonological information is not the critical one in determining whether a concurrent memory load will interfere with temporal processing.

\section{EXPERIMENT 3}

The results of Experiment 2 indicated that the noninterference with interval production by a phonological memory load found by Fortin and Breton (1995) and Fortin and Massé (1999) is not a unique phenomenon. The aim of Experiment 3 was to establish whether the interference produced by varying a pitch memory load in Experiment 1 was due to a unique interaction between the pitch memory system and the pacemaker-counter system, or whether the interaction is a more general one between temporal processing and auditory memory. To address this question, the conditions of Experiment 1 were replicated using a different auditory short-term memory task, requiring memory for timbre, which is that quality of a sound that distinguishes it from another sound of identical pitch and intensity. The main advantage of using a 


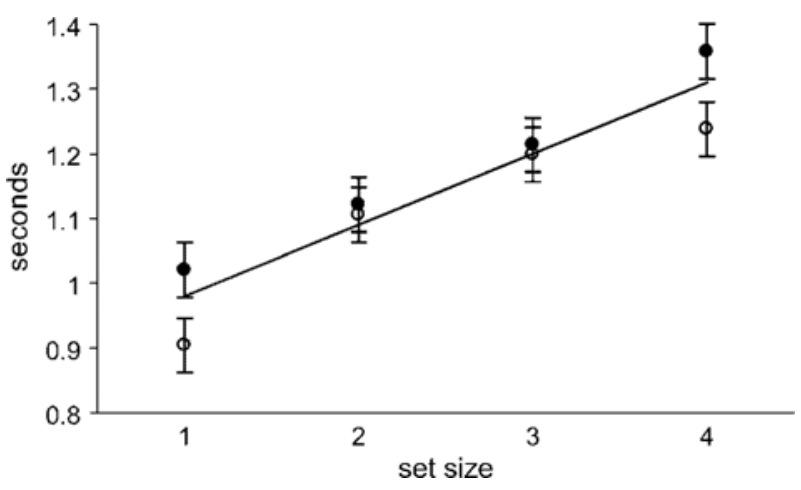

Figure 5. The effect of set size and whether the probe item was present (open circles) or absent (filled circles) from the color memory set on reaction time when the probe item was presented after time production was complete, together with the best linear fit to the data points.

timbre-based memory task was that the pitch of the memory items could be held constant. Therefore, there was no pitch memory load, and any set size effect that occurred could not be due to an uncontrolled pitch component in the task. Furthermore, evidence reviewed briefly below suggests that there is a functional dissociation between short-term memory for pitch and short-term memory for timbre. Therefore, if a set size slope occurred, this would suggest a general interaction between the pacemakercounter system and auditory short-term memory. However, if no set size slope occurred, this would suggest that the interaction is highly specific to pitch memory.

Within auditory short-term memory, a functional dissociation has been established between memory for pitch and memory for timbre. The paradigm used by Deutsch (1970) to establish the dissociation between pitch memory and phonological memory has also been used to establish the separation between pitch and timbre. Semal and Demany (1991) demonstrated that varying the timbre of material interpolated in a retention interval had no impact upon pitch recognition judgments. Starr and Pitt (1997) showed that recognition memory for timbre is sensitive to interference from the timbre of intervening stimuli and that varying the pitch of intervening stimuli has no impact upon timbre recognition.

Subjective pitch is a simple function of physical frequency. The determination of subjective timbre is more complex. Various attributes of a sound can affect timbre, and these factors may interact with each other. These factors include the attack and decay times of various components of a sound, as well as the spectral composition. For the purposes of the present experiment, varying the attack and decay times was undesirable because this would encourage the subjects to use temporal cues to distinguish the memory set items from each other. This would create a situation in which two temporal tasks were being performed concurrently, making interference extremely likely to occur. What happens when two temporal tasks are combined was addressed in Experiment 4.
Fortunately, the spectral composition of a sound may be varied while attack and decay times are kept constant, resulting in a systematically varying percept without temporal differences resulting. This method of varying timbre was used by Semal and Demany (1991) and Starr and Pitt (1997).

For the present experiment, a memory set composed of eight items was generated by varying the spectral composition of a tone with a fixed fundamental frequency.

\section{Method}

Subjects. Twelve subjects, between 21 and 33 years of age, took part in the main experiment. As an incentive, the 3 subjects with the lowest error rates were awarded cash prizes. Twelve unpaid subjects, between 21 and 45 years of age, took part in the similarity judgment task.

Stimuli. All of the eight tones in the timbre memory set were based on a fundamental frequency of $200 \mathrm{~Hz}$. Therefore, they were identical to each other in pitch. Each tone had two harmonic components. The frequencies of the harmonics were systematically varied in order to produce a range of stimuli that sounded like different voices of an early electronic synthesizer. However, the sounds were all subjectively close to their immediate neighbors, and there was no categorical distinction between any of the sounds. The physical steps in the harmonics were equal from tone to tone. Thus, the first tone consisted of the fundamental plus the first and second harmonics, the second tone consisted of the fundamental plus the fourth and fifth harmonics, the third tone consisted of the fundamental plus the seventh and eighth harmonics, and so forth. The amplitude (intensity) of each of the two harmonics was equal to that of the fundamental. The stimuli were presented using the same headphones as those in Experiment 1, and the stimulus intensity was set in the same way as in Experiment 1.

Procedure. Each memory set item was presented for $500-\mathrm{msec}$, with 500-msec pauses between items, as in Experiment 1. The rest of the procedure was identical to that in Experiment 2 and the equivalent condition in Experiment 1.

\section{Results}

Timbre memory load concurrent with interval production. The overall error rate in the memory search component of the task was $18.7 \%$, as compared with $17.6 \%$ in the equivalent condition in Experiment 1 . The details of this are presented in Table 3, from which it can be seen that the error rate increased with memory set size. After trials on which a memory search error occurred were excluded, outlying data points were removed. This amounted to $1.44 \%$ of the remaining memory search data and $0.32 \%$ of the remaining interval production data.

In the final block of interval production practice without feedback, the mean interval produced was $2,570 \mathrm{msec}$, and the mean time produced in the experimental blocks

Table 3

Percentage Error Rates for the Timbre Memory Search Task in Experiment 3

\begin{tabular}{ccccc}
\hline & \multicolumn{4}{c}{ Memory Set Size } \\
\cline { 2 - 5 } Probe Type & 1 & 2 & 3 & 4 \\
\hline Positive & 9.0 & 15.6 & 20.5 & 24.3 \\
Negative & 9.7 & 19.1 & 19.4 & 31.9 \\
\hline
\end{tabular}


$(2,558 \mathrm{msec})$ did not differ reliably from this. Figure 4 shows the relationship between the number of items in the memory set and interval production. The regression slope of set size with time production was $9 \mathrm{msec}$. A linear contrast indicated that this small slope was not significant $[F(1,11)=1.28$, n.s. $]$.

Response times to the memory probe, which was presented after interval production was complete, are shown in Figure 6. The linear contrast for memory set size was significant $[F(1,11)=13.82, p<.01]$. The regression slope of the function was $41 \mathrm{msec}$ per extra item, which was slightly less than that found for pitch in Experiment 1 .

\section{Discussion}

The task difficulty of the timbre memory search task, as assessed by the error rate, was similar to that of the pitch memory search task.

In contrast to the positive result with pitch in Experiment 1 , increasing the number of timbre items to be maintained during interval production had no effect on interval production. Because the two experiments were identical to each other in every respect, apart from the pitch/timbre manipulation, these results suggest that a very specific resource is shared by short-term memory for pitch and the pacemaker-counter system. Whatever this resource is, it is not required by the maintenance of phonological material, color, or timbre.

\section{EXPERIMENT 4}

It was established in Experiments 1-3 that maintenance of pitch in short-term memory produces a specific and systematic interference with interval production. This result qualifies the conclusion of Fortin and Breton (1995) that only active processing of information in shortterm memory interferes with interval production, although this conclusion is correct for phonological mate-

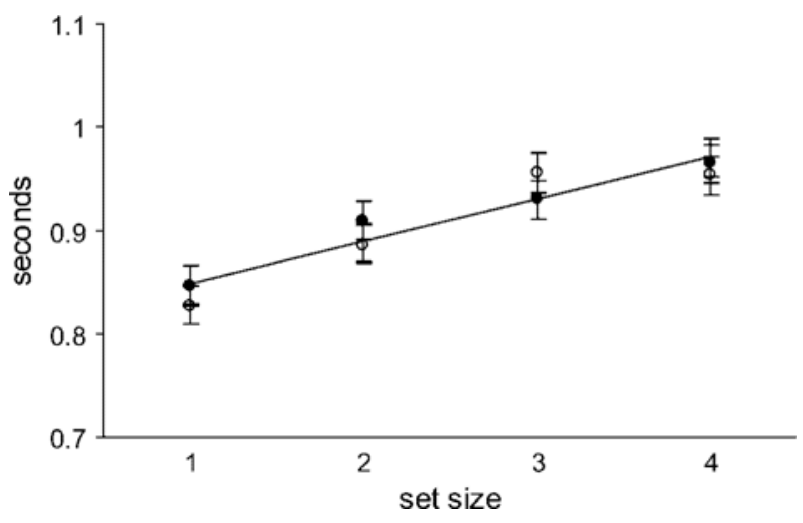

Figure 6. The effect of set size and whether the probe item was present (open circles) or absent (filled circles) from the timbre memory set on reaction time when the probe item was presented after time production was complete, together with the best linear fit to the data points. rial, color, and timbre. Experiment 4 sought to produce interference with the pacemaker-counter system during interval production by employing a secondary task that was itself dependent on the operation of part of the temporal processing system.

The pacemaker-counter theory assumes that the value in the counter can be maintained within a short-term memory system. This is necessary to enable the value in the counter to grow. In order to account for experimental findings, such as those of Fortin and Massé (2000), it is also necessary to propose that count values can be retained accurately in short-term memory over a period of at least a few seconds. Fortin and Massé (2000) included breaks of up to $6,000 \mathrm{msec}$ in a 2,000 -msec interval production. Part of the way through interval production, a signal indicated that the subjects were to interrupt their production of the interval. After the break was complete, a signal indicated that interval production was to continue. There was no effect of break duration on the mean interval production. This suggests that short-term memory can maintain the counter value when the counter is stopped, as well as the value for the eventual target. It also rules out memory decay as a source of inaccuracy in interval production, at least over periods of a few seconds. Similar results to those in Fortin and Massé (2000) were obtained by Roberts (1981), who required rats trained on longer target durations to cope with substantial breaks in the timed interval. Because decay does not seem to be a major factor in short-term memory for duration, we chose to investigate the possibility that short-term memory for duration is subject to interference from other memory tasks requiring temporal processing. Experiments 1-3 reported here, in conjunction with the results of Fortin and Breton (1995), suggest that interference with short-term memory for duration from other nontemporal memory tasks is negligible, except in the unusual case of pitch memory. Here, we required the subjects to produce the 2,500-msec target interval, while also maintaining a number of duration values in short-term memory for a subsequent recognition test. Thus, the experimental paradigm was the same as that used in Experiments 1-3, but the secondary task involved remembering values of duration. Both the primary and the secondary tasks were assumed to rely upon the short-term memory system that maintains counter values. It was also assumed that the temporal short-term memory system has a capacity limit, and therefore interference was predicted.

A pilot experiment was conducted to establish appropriate values of duration for use in the memory set. With a memory set in which each item's duration was double that of the one before it, a relatively steep reaction time slope of $114 \mathrm{msec}$ per item was obtained, with an error rate of $25.9 \%$. Because of the high error rate, only set sizes 1-3 were employed in the main experiment. As a result of the doubling procedure, the memory set items varied widely in duration, from 18 to $2,304 \mathrm{msec}$. This provided the opportunity to answer a further question: Would interference be related to the number of items in 
memory (as was the case for pitch) or to the total magnitude of duration that had to be maintained on a given trial?

\section{Method}

Subjects. Thirteen subjects, between 18 and 33 years of age, took part in the experiment. They received course credit in return for their participation.

Stimuli. All of the stimuli used were $2000-\mathrm{Hz}$ pure tones, presented using headphones, at an intensity slightly below the comfortable maximum (approximately 70 decibels). The values of duration used for the eight items in the memory set were $18,36,72$, $144,288,576,1,152$, and 2,304 msec. During the presentation of memory sets, individual items were separated by $576-\mathrm{msec}$ pauses.

The conditions for responding to the probe item, which was presented after interval production was complete, were slightly different from those for the stimuli in Experiments 1-3. Specifically, the subjects were instructed not to respond until the probe item ceased to sound (in the earlier experiments, their response terminated the presentation of the probe item). Therefore, response time was measured from the offset of the probe item. Memory sets and probe items were selected in the same way as for the previous experiments. However, trials on which the probe item was negative and two or more steps longer than the longest set member were excluded from the analysis of the response time data only. ${ }^{1}$ This prevented trials in which it was possible to make the recognition decision prior to probe offset, resulting in an artificially fast response time, from contributing to the results.

Procedure. The interval production training procedure was identical to that used in the earlier experiments. There were four blocks of 48 experimental trials. Therefore, each subject received 64 trials in each interval production condition of the experiment, which resulted in 32 for each reaction time condition. This number was greater than those in Experiments 1-3 because a higher error rate was anticipated

\section{Results}

The overall error rate in the memory search component of the task was $27.3 \%$. The details of this are presented in Table 4. After trials on which a memory search error occurred were excluded, outlying data points were removed. This amounted to $2.2 \%$ of the remaining memory search data and $1.5 \%$ of the remaining interval production data.

In the final block of interval production practice without feedback, the mean interval produced was 2,666 msec, and the mean interval produced in the experiment $(2,572 \mathrm{msec})$ did not differ significantly from this. Figure 7 shows the relationship between the number of items in the memory set and interval production. The regression slope of time production with set size was $60 \mathrm{msec}$, as compared with $26 \mathrm{msec}$ for the equivalent pitch condition in Experiment 1. The linear contrast for this effect was highly significant $[F(1,12)=22.80, p>.001]$.

Table 4

Percentage Error Rates for the Duration Memory Search Task in Experiment 4

\begin{tabular}{cccc}
\hline & \multicolumn{3}{c}{ Memory Set Size } \\
\cline { 2 - 4 } Probe Type & 1 & 2 & 3 \\
\hline Positive & 11.8 & 23.7 & 23.7 \\
Negative & 25.4 & 39.2 & 40.2 \\
\hline
\end{tabular}

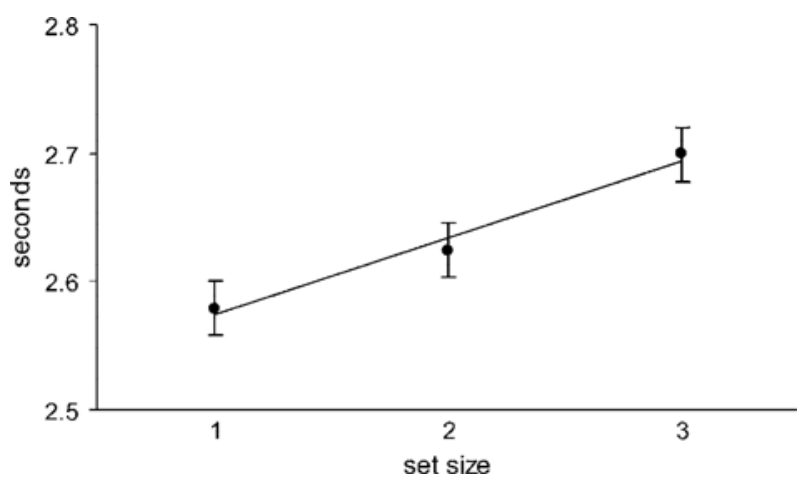

Figure 7. The effect of increasing the number of items to be remembered concurrently with time production when the critical feature of the memoranda is their duration.

Response times to the memory probe, which was presented after interval production, are shown in Figure 8. The linear contrast of memory set size was significant $[F(1,12)=13.99, p>.01]$, and the effect of probe type (i.e., in or out of the memory set) was also highly significant $[F(1,12)<13.07, p<.01]$. The reaction time slope was $148 \mathrm{msec}$ per item, as compared with $114 \mathrm{msec}$ for the equivalent conditions in the reaction time pilot experiment.

The possibility that interference with interval production would be related to the total duration of the items concurrently maintained was assessed using a correlational approach. First, the total duration of each memory set was calculated by simply adding up the values, in milliseconds, of the individual items. The resulting variable was correlated with values of interval production from corresponding trials, for each subject separately. The correlation was significant for only 3 of the 14 subjects, and in each of these cases the value of Pearson's $r$ was less than .25. This suggests that the total duration of the items was of less importance in determining the extent of interference with interval production than was the number of items being retained.

\section{Discussion}

The requirement to remember values of duration while also producing a learned interval biased the subjects toward producing increasingly longer intervals as the number of items in the concurrent memory load was increased. There was very little evidence that the total duration remembered determined the extent of biasing. This pattern of results suggests that the capacity limit of short-term memory for duration is item based, rather than total duration based. This implies that the values in the counter are like a count in the sense that they are a nontemporal representation of time. If actual durations were kept on line by repeatedly running neural processes with durations equal to the values to be kept active in memory, the capacity limit would have manifested itself in terms of total duration, as well as the number of items. The present experiment did not set out to quantify the capacity limit, but it is clear from the error rates that sub- 


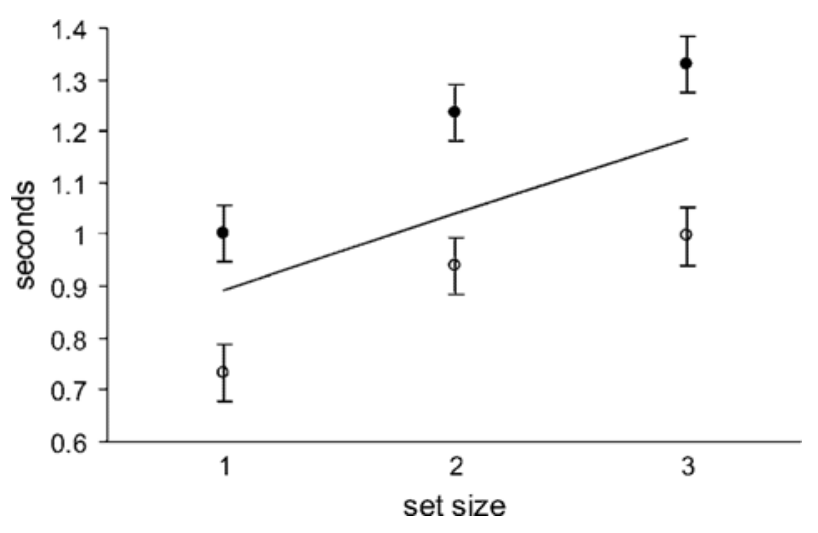

Figure 8. The effect of set size and whether the probe item was present (open circles) or absent (filled circles) from the duration memory set on reaction time when the probe item was presented after time production was complete, together with the best linear fit to the data points.

jects have trouble representing more than two durations (one in memory and a comparison duration) at a time.

The magnitude of the increase in interval production produced by the duration-based secondary task was similar to that produced by retaining pitch information in Experiment 1. Because the duration-based secondary task was deliberately designed to employ processing resources also involved in interval production, whereas there is no such obvious overlap in the case of the pitch task, it was surprising that the interference from the duration-based secondary task was not greater.

\section{GENERAL DISCUSSION}

Fortin and Breton (1995) suggested that secondary tasks interfere with temporal processing to the extent that they require the active manipulation of material in short-term memory. The data presented here demonstrate that, in some cases, a secondary task that requires only maintenance of material in short-term memory can cause disruption of timing of a similar nature to that caused by the active short-term memory tasks used by Fortin et al. (1993). Whether interference occurs is unrelated to the difficulty level of the secondary memory task, but it is related to the kind of memoranda used.

The error rates reported here are fairly high for the higher memory set sizes, especially where the memory probe item was presented after interval production. As the error rate rises, the number of trials on which the subject pressed the correct button in response to the memory probe by successfully guessing, as opposed to making a genuine response, increases. Because there is no way of estimating the duration of a guessed response, the influence of guessing on the response time slopes reported here is unknown. However, one reassuring comparison may be made. When the pitch memory probe was presented after interval production in Experiment 1, this resulted in a much higher error rate than that in the reaction time condition. However, the slopes of response time with set size were similar- $60 \mathrm{msec}$ versus $66 \mathrm{msec}$. It is also worth noting that the error rate in the memory search component of Fortin and Breton's (1995) experiment in which digits were retained during interval production was double that for the condition in which the probe item was presented during interval production in Fortin et al. (1993). In the case of interval production where the subject subsequently makes a "correct" guessed response to the memory probe, it is not certain that all the items were genuinely retained during interval production. However, the error rates for the pitch, color, and timbre experiments in which the memory search was performed after interval production were similar to each other. Therefore, whatever the influence of the error rate was on the function of interval production with number of items retained, errors should not affect the comparisons between these three experiments.

A key issue for the interpretation of the present results is that of the cognitive requirements of the secondary tasks. It is assumed that comparing a probe item with a set of items held in short-term memory is an active process. In most of the experiments reported here, this active process was isolated from time production by having it performed after time production was complete. It is assumed that the processes involved in storing the set of items are more passive in nature. This distinction was made by Fortin and Breton (1995) on the basis that, in the case of digits, the former kind of processing produced a systematic load-related interference with time production, whereas the small amount of interference from storage only was not load related. Indeed, it has been argued by Fortin, Duchet, and Rousseau (1996) that errors in timing are a sensitive probe of the loading of short-term memory by a concurrent task. Here, passive retention of pitch information produced a load-related effect on timing, in contrast to the lack of a load-related effect for timbre and color reported here and for digits by Fortin and Rousseau (1998). One possibility that should be considered is that the unusual result for pitch occurred because the processing involved in the storage of pitch is more active than that required by the storage of the other memoranda.

This possibility is suggested by the findings of Krumhansl and Iverson (1992, Experiments 2 and 3), whose subjects made judgments of the pitch or timbre of tones embedded within sequences of tones. Their results showed that pitch was perceived in relation to the surrounding context tones, whereas timbre was not. This result confirmed experimentally that a set of pitches can produce a kind of gestalt, normally referred to as melody, whereas timbre does not produce a comparable gestalt. If the pitch memory sets in the experiments reported here were being perceived as melodies, it may be suggested that their storage required an additional processing step, over and above those of the other memory sets that did not produce interference with temporal processing. However, the tones used here were quite different from those used by 
Krumhansl and Iverson, in the critical sense that they were chosen to avoid the possibility of melody, as the relation between the pitches used did not correspond to any Western musical scale. Krumhansl and Iverson's tones were chosen to span a single octave of the octatonic musical scale. It is, therefore, an open question whether their results could be replicated with the set of tones employed here.

In the case of interference with interval production caused by retaining pitch and duration information, it is not clear what the mechanism of interference is. Where the memory probe item is processed during interval production, as for pitch in one of the conditions of Experiment 1 and for digits in Fortin et al. (1993), the gate theory can provide an explanation; resources normally used to keep the gate closed during timing, so that pulses can pass from the pacemaker to the accumulator, are temporarily redirected toward memory search. This creates an interruption in the process of accumulating pulses toward a target value, which is proportional to the duration of memory search. Where memory items are retained only during interval production, it is hard to see when the gate would operate to produce an interruption. Nonetheless, interval production does lengthen with set size, which suggests that the time taken for the pulses in the accumulator to equal the target value is increased by the presence of certain memory loads. This might occur if the pacemaker was slowed down or the short-term memory store supporting the counter was disrupted. The latter possibility seems particularly likely in the case of disruption from a concurrent duration memory load, but the present data do not provide a test of this idea.

\section{REFERENCES}

BADDEley, A. D. (1986). Working memory. Oxford: Oxford University Press.

Brown, S. W. (1997). Attentional resources in timing: Interference effects in concurrent temporal and nontemporal working memory tasks. Perception \& Psychophysics, 59, 1118-1140.

Brown, S. W., \& WeST, A. N. (1990). Multiple timing and the allocation of attention. Acta Psychologica, 75, 103-121.

CASINI, L., \& MACAR, F. (1997). Effects of attention manipulation on judgments of duration and of intensity in the visual modality. $\mathrm{Mem}-$ ory \& Cognition, 25, 812-818.

CRUSE, D., \& JoNES, R. A. (1976). Intentional forgetting of tones in a choice recognition-time task. Journal of Experimental Psychology: Human Learning \& Memory, 2, 577-585.

Deutsch, D. (1970). Tones and numbers: Specificity of interference in short-term memory. Science, 168, 1604-1605.

DeutsCH, D. (1972). Mapping of interactions in the pitch memory store. Science, 175, 1020-1022.

Deutsch, D. (1975). Facilitation by repetition in recognition memory for tonal pitch. Memory \& Cognition, 3, 263-266.

Deutsch, D., \& Feroe, J. (1975). Disinhibition in pitch memory. Perception \& Psychophysics, 17, 320-324.

Fetterman, J. G., \& Killeen, P. R. (1990). A computational analysis of pacemaker-counter timing systems. Journal of Experimental Psychology: Human Perception \& Performance, 16, 766-780.
Fortin, C., \& Breton, R. (1995). Temporal interval production and processing in working memory. Perception \& Psychophysics, $\mathbf{5 7}$, 203-215.

Fortin, C., Duchet, M. L., \& Rousseau, R. (1996). Tapping sensitivity to processing in short-term memory. Canadian Journal of Experimental Psychology, 50, 402-407.

ForTIN, C., \& MASSÉ, N. (1999). Order information in short-term memory and time estimation. Memory \& Cognition, 27, 54-62.

ForTin, C., \& Massé, N. (2000). Expecting a break in time estimation: Attentional time-sharing without concurrent processing. Journal of Experimental Psychology: Human Perception \& Performance, 26 1788-1796.

Fortin, C., \& Rousseau, R. (1998). Interference from short-term memory processing on encoding and reproducing brief durations. Psychological Research, 61, 269-276.

Fortin, C., Rousseau, R., Bourque, P., \& Kirouac, E. (1993). Time estimation and concurrent nontemporal processing: Specific interference from short-term-memory demands. Perception \& Psychophysics, 53, 536-548.

Gibbon, J., CHurch, R. M., \& Meck, W. H. (1984). Scalar timing in memory. In J. Gibbon \& L. G. Allan (Eds.), Timing and time perception (Annals of the New York Academy of Sciences, Vol. 423, pp. 5257). New York: New York Academy of Sciences.

Hicks, R. E., Miller, G. W., \& Kinsbourne, M. (1976). Prospective and retrospective judgments of time as a function of amount of information processed. American Journal of Psychology, 89, 719-730.

Krumhansl, C. L., \& Iverson, P. (1992). Perceptual interactions between musical pitch and timbre. Journal of Experimental Psychology: Human Perception \& Performance, 18, 739-751.

Peretz, I., Belleville, S., \& Fontaine, S. (1997). Dissociations entre musique et langage après atteinte cerebrale: Un nouveau cas d'amusie sans aphasie [Dissociations between music and language after cerebral damage: A new case of music deficits without aphasia]. Canadian Journal of Experimental Psychology, 51, 354-367.

Peretz, I., Kolinsky, R., Tramo, M., Labreque, R., Hublet, C., Demeurisse, G., \& Belleville, S. (1994). Functional dissociations following bilateral lesions of auditiory cortex. Brain, 177, 1283-1301.

RoBERTS, S. (1981). Isolation of an internal clock. Journal of Experimental Psychology: Animal Behavior Processes, 7, 242-268.

Semal, C., \& Demany, L. (1991). Dissociation of pitch from timbre in auditory short-term memory. Journal of the Acoustical Society of America, 89, 2404-2410.

StARR, G. E., \& PitT, M. A. (1997). Interference effects in short-term memory for timbre. Journal of the Acoustical Society of America, 102, 486-494.

STERNBERG, S. (1966). High-speed scanning in human memory. Science, 153, 652-654.

ZAKAY, D. (1989). Subjective time and attentional resource allocation: An integrated model of time estimation. In I. Levin \& D. Zakay (Eds.), Time and human cognition: A life span perspective (pp. 365397). Amsterdam: Elsevier.

ZAKAY, D. (1993). Time estimation methods: Do they influence prospective duration estimates? Perception, 22, 91-101.

ZaKaY, D., \& Block, R. A. (1997). Temporal cognition. Current Directions in Psychological Science, $\underline{6}_{2}$ 12-16.

\section{NOTE}

1. This resulted in the removal of $38.2 \%, 19.2 \%$, and $12.5 \%$ of the data at set sizes 1,2 , and 3 for the negative probe items only.

(Manuscript received September 23, 2002; revision accepted for publication October 16, 2003.) 